\title{
Cathodoluminescence and Raman Spectromicroscopy of Forsterite in Tagish Lake Meteorite: Implications for Astromineralogy
}

\author{
Arnold Gucsik, ${ }^{1,2}$ Ildikó Gyollai, ${ }^{2,3}$ Hirotsugu Nishido, ${ }^{4}$ \\ Kiyotaka Ninagawa, ${ }^{5}$ Matthew M. R. Izawa, ${ }^{6}$ Cornelia Jäger, ${ }^{7}$ Ulrich Ott, ${ }^{8}$ \\ Irakli Simonia, ${ }^{9}$ Szaniszló Bérczi, ${ }^{3}$ and Masahiro Kayama ${ }^{10}$ \\ ${ }^{1}$ Department of Geology, University of Johannesburg, Auckland Park, Johannesburg 2600, South Africa \\ ${ }^{2}$ Konkoly Thege Miklós Astronomical Institute, Research Centre for Astronomy and Earth Sciences, Hungarian Academy of Sciences, \\ Konkoly Thege Miklós út 15-17, Budapest 1121, Hungary \\ ${ }^{3}$ Cosmic Material Space Research Group, Institute of Physics, Department of Material Physics, Faculty of Science, \\ Eötvös Loránd University, Pázmány Péter Sétány 1/a, Budapest 1117, Hungary \\ ${ }^{4}$ Department of Biosphere-Geosphere System Science, Okayama University of Science, 1-1 Ridai-cho, Okayama 700-0005, Japan \\ ${ }^{5}$ Department of Applied Physics, Okayama University of Science, 1-1 Ridai-cho, Kita-ku, Okayama 700-0005, Japan \\ ${ }^{6}$ University of Winnipeg, 515 Portage Avenue, Winnipeg, MB, Canada R3B 2E9 \\ ${ }^{7}$ Laboratory Astrophysics Group, The Max Planck Institute for Astronomy, Institute of Solid State Physics, \\ The Friedrich Schiller University Jena, Helmholtzweg 3, 07743 Jena, Germany \\ ${ }^{8}$ Savaria University Center, University of West Hungary, Károlyi Gáspár tér 4, Szombathely 9700, Hungary \\ ${ }^{9}$ School of Graduate Studies, Ilia State University, Kakutsa Cholokashvili Avenue 3/5, 0162 Tbilisi, Georgia \\ ${ }^{10}$ Department of Earth and Planetary Sciences, Faculty of Science, Kobe University, 1-1 Rokkodai-cho, Nada-ku, Kobe 657-8501, Japan
}

Correspondence should be addressed to Arnold Gucsik; argu1986@hotmail.com

Received 31 October 2015; Accepted 28 January 2016

Academic Editor: Jin Zhang

Copyright (c) 2016 Arnold Gucsik et al. This is an open access article distributed under the Creative Commons Attribution License, which permits unrestricted use, distribution, and reproduction in any medium, provided the original work is properly cited.

\begin{abstract}
The Tagish Lake meteorite is CI/CM2 chondrite, which fell by a fireball event in January 2000. This study emphasizes the cathodoluminescence (CL) and Raman spectroscopical properties of the Tagish Lake meteorite in order to classify the meteoritic forsterite and its relation to the crystallization processes in a parent body. The CL-zoning of Tagish Lake meteorite records the thermal history of chondrules and terrestrial weathering. Only the unweathered olivine is forsterite, which is CL-active. The variation of luminescence in chondrules of Tagish Lake meteorite implies chemical inhomogeneity due to low-grade thermal metamorphism. The blue emission center in forsterite due to crystal lattice defect is proposed as being caused by rapid cooling during the primary crystallization and relatively low-temperature thermal metamorphism on the parent body of Tagish Lake meteorite. This is in a good agreement with the micro-Raman spectroscopical data. A combination of cathodoluminescence and micro-Raman spectroscopies shows some potentials in study of the asteroidal processes of parent bodies in solar system.
\end{abstract}

\section{Introduction}

The Tagish Lake meteorite fell on 18th January, 2000, at 16:43 UTC [1]. Tagish Lake fireball event ended with airburst explosions near Carcross, Yukon Territory, Canada, and debris crossed the Yukon-British Columbia boundary, with many fragments finally landing on Taku Arm of Tagish Lake, British
Columbia, Canada $\left(59^{\circ} 42^{\prime} \mathrm{N}, 134^{\circ} 12^{\prime} \mathrm{W}\right)$. The original preatmospheric mass of the Tagish Lake meteorite was estimated at 200,000 [1], 56,000 [2], and 60,000-90,000 kg [3]. Tagish Lake was initially classified as the first CI2 chondrite [4] but has subsequently been regarded as an ungrouped C2 chondrite with affinities to both CM and CI chondrites. Out of the $10 \mathrm{~kg}$ recovered in total, about $870 \mathrm{~g}$ was collected within 
about a week after the fall, on 25th-26th January, 2000, from the frozen lake surface [3]. The Tagish Lake meteorite is a brecciated, matrix-dominated material with multiple lithologies [5-9]. Tagish Lake contains sparse olivine-dominated chondrules generally of less than $1 \mathrm{~mm}$ diameter, altered CAIs of up to $2 \mathrm{~mm}$ diameter, magnetite, individual grains of olivine, $\mathrm{Ca}-\mathrm{Fe}-\mathrm{Mn}$ carbonates (calcite and siderite-magnesite with rarer dolomite), and $\mathrm{Fe}-\mathrm{Ni}$ sulfides including pyrrhotite (e.g., $[1,5,6])$. The chondrule mesostasis glass material is partially replaced by phyllosilicates indicative of aqueous alteration [10]. Most CAIs are completely altered to phyllosilicates (mainly Mg-rich serpentine, saponite) and carbonates (dolomite, calcite), though some retain spinel-dominant primary material [6]. Tagish Lake matrix is mineralogically similar to that of other aqueously altered carbonaceous chondrites (CM, CI, and CR chondrites) and is composed of Mgphyllosilicates, fine-grained Fe-Ni sulfides, magnetite, and Fe-Mg carbonates (e.g., $[5,7,10])$. Tagish Lake meteorite is an unshocked (S1) type $[1,6]$, like most carbonaceous chondrites $[1,11]$. Herd et al. [10] identified carboxylic acids, amino acids, and aliphatic and aromatic hydrocarbons in organic fractions. Brown et al. [1] interpreted Tagish Lake meteorite low-temperature (perhaps approaching $\sim 0^{\circ} \mathrm{C}$ ) aqueous alteration to have taken place on a water-bearing parent asteroid, similar to the case of other aqueously altered carbonaceous chondrites, while Hiroi et al. [12] proposed the Tagish Lake meteorite as the first sample of a D-type asteroid. Mittlefehldt [13] concludes that the elemental composition of Tagish Lake is generally closer to CM chondrites than to CI but also found that some of the most volatile elements were higher than in CM. The carbon content, according to Grady et al. [4], is $5.81 \mathrm{wt} \%$, which is higher than typical concentrations for CM or CI carbonaceous chondrites. Carbon in Tagish Lake occurs in carbonate minerals [5], various organic compounds, and nanodiamonds (e.g., [4, 10]). Brown et al. [1] measured oxygen isotopic compositions close to CI chondrites. Grady et al. [4] observed higher concentrations of presolar nanodiamonds in Tagish Lake than in any other meteorite. Mittlefehldt [13] concluded, based primarily on the apparent higher concentrations of presolar nanodiamonds (stardust grains), that the Tagish Lake parent body accreted at larger heliocentric distances than most other carbonaceous chondrites. The organic material in Tagish Lake meteorite was studied by numerous groups including Pizzarello et al. [14] and Nakamura-Messenger et al. [15], who proposed that the organic globules in Tagish Lake material originated from protoplanetary disk.

\section{Samples and Experimental Procedure}

Our Tagish Lake sample consisted of two thin sections, which are embedded in epoxy resin on a glassy slide. Samples for this study are from Spring 2000 (nonpristine) collection and originate from collection site MG-02 $[1,13]$. The sections were prepared by hand using isopropyl alcohol as the only solvent and were set in Struers EpoFix epoxy. The same sections were studied by Izawa et al. [6]; see their Figures 3 C-D, 4, and 8. One area has been selected from each of the slides, each containing forsterite-rich chondrules in fine-grained matrix.
Chondrule olivine in Tagish Lake is dominantly forsteritic in composition, with $\mathrm{Ca}, \mathrm{Cr}$, and $\mathrm{Mn}$ as the most common minor elements (e.g., $[6,16])$. Chondrule olivine in the thin section studied here (the same as that used by Izawa et al. [6]) has an average $\mathrm{Cr}_{2} \mathrm{O}_{3}$ content of $0.30 \mathrm{wt} \%$ and $\mathrm{MnO}$ content of $0.03 \mathrm{wt} \%$ [6]. The grains are chemically homogeneous in SEM X-ray map [6] and are compositionally close to endmember forsterite (average chondrule olivine composition $\mathrm{Fo}_{98.9}$ for this section, Izawa et al. [6], their Table 2). CL color imaging was obtained using a luminoscope (ELM-3R) with a cooled charge-coupled device (CCD) camera, which was operated with electron beams generated by excitation voltage at $10 \mathrm{kV}$ and beam current of $0.5 \mathrm{~mA}$. This system also contains a cold cathode discharge tube and a vacuum chamber. The diameter of electron beam spot at a few $\mathrm{mm}$ size on the sample surface was controlled by a magnet. The Nikon imaging system (DS-5Mc) was used to convert CL images to digital data.

JSM-5410LV Scanning Electron Microscope (SEM) consisting of MiniCL detector with a multialkali photomultiplier tube was used to obtain CL scanning images at high magnification. A grating monochromator of the SEM-CL facility was used with the following operating conditions: 1200 grooves $/ \mathrm{mm}$, a focal length of $0.3 \mathrm{~m}, \mathrm{~F}$ of 4.2 , limit of resolution of $0.5 \mathrm{~nm}$, and slit width of $4 \mathrm{~mm}$ at the inlet and outlet. CL spectral data were recorded by a photon counting method using a photomultiplier tube (Hamamatsu, R2228) and converted to digital data.

Further details of the CL equipment and analytical procedure can be seen in Kayama et al. [17]. The OriginPro 8J SR2 software containing a peak fitting option (peak analyzer) was used for the correction and deconvolution of each emission center.

Micro-Raman analyses were carried out using the LabRam Confocal Spectrometer (632 nm excitation) of the Laboratory Astrophysics Group of the Max Planck Institute for Astronomy (Jena, Germany). Single spectrum was acquired with an integration time of $30 \mathrm{sec}$ at 3 accumulations, and spectral range of $100-4400 \mathrm{~cm}^{-1}$ and $20 \mathrm{x}$ and $50 \mathrm{x}$ objectives were used for the collection of Raman spectra. Here, we present Raman results selecting the most representative Raman spectrum.

\section{Results}

3.1. Optical Microscopy. The studied area contains a strongly aqueous-altered porphyritic chondrule $(\sim 800 \mu \mathrm{m}$ diameter $)$, which has a fine-grained accretionary rim. The formerly glassy chondrule mesostasis has been replaced by phyllosilicates, which has been discernible in the optical images (Figures $1(\mathrm{a})$ and $1(\mathrm{c}))$. The individual olivine phenocrysts range is $10-50 \mu \mathrm{m}$ in the longest dimension, but a large $300 \mu \mathrm{m}$ amoeboid olivine aggregate occurs in the chondrule. The second chondrule is less altered, which is an intermediate between granular (TLa left side of chondrule) and barred texture (TLa right-side of chondrule). The mesostasis has been altered to a phyllosilicate-dominant material. In both chondrules the olivine crystals show zoned structure, which may correspond to structural inhomogeneity or lattice strain. The area TLb 


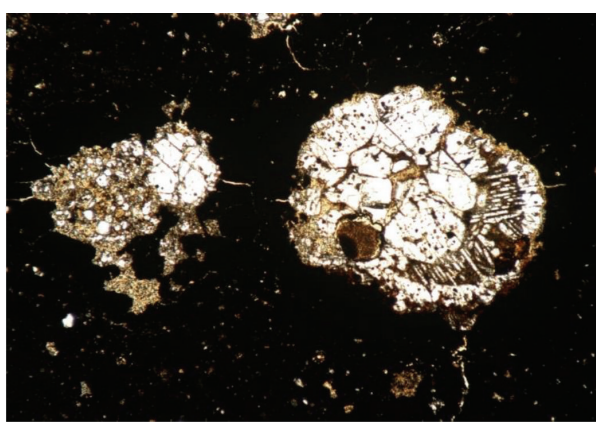

(a)

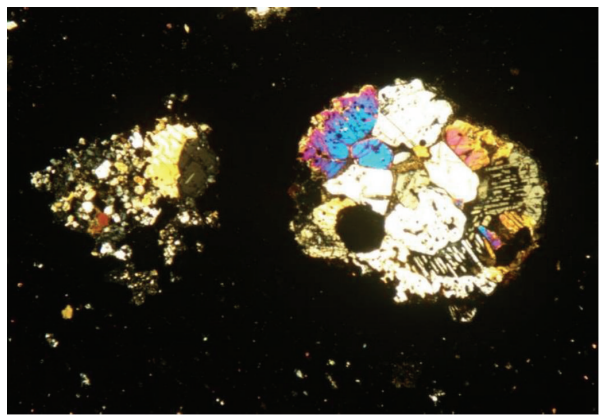

(c)

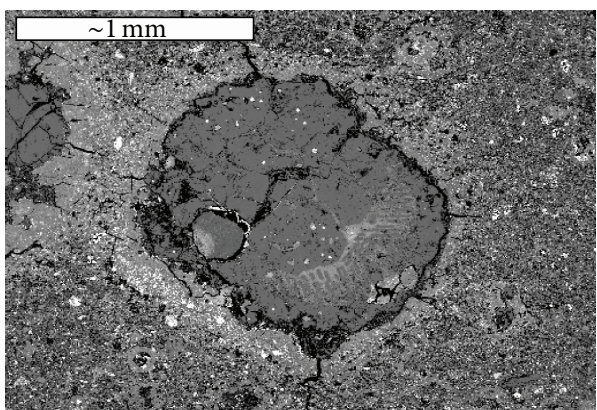

(e)

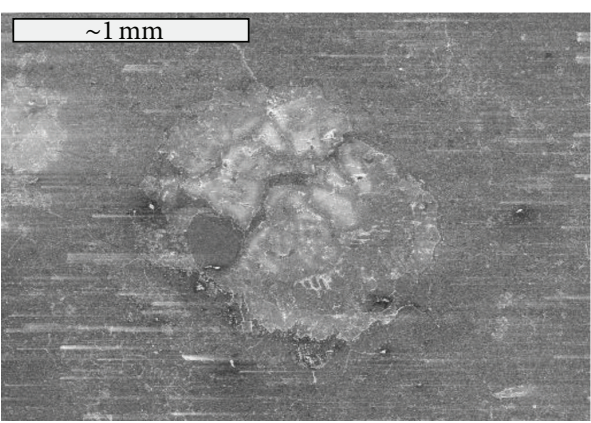

(b)

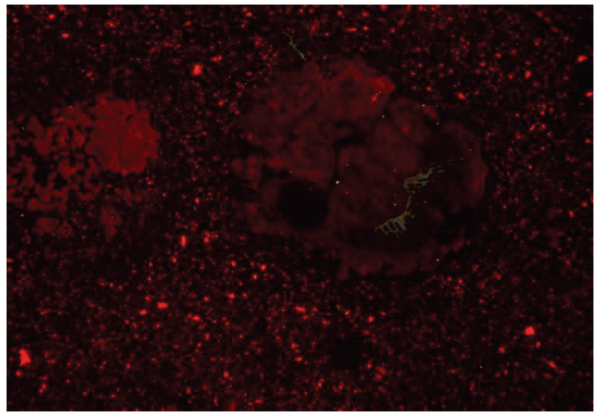

(d)

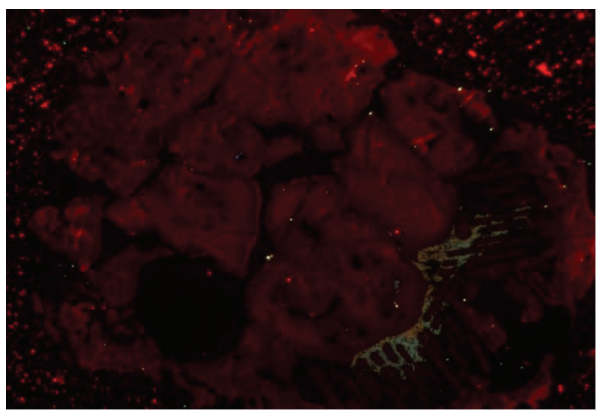

(f)

Figure 1: Optical microscope ((a) reflected light and (c) cross-polarized modes), scanning electron microscope ((e) backscattered electron microscope and (b) cathodoluminescence), and cathodoluminescence color ((d) and (f) at high magnification of the selected grain) images of TLa area showing a forsteritic barred chondrule. Scale: width of the chondrule is around $1 \mathrm{~mm}$.

is composed of mineral-fragment rich groundmass, which contains a strongly altered forsterite chondrule (Figures 2(a) and $2(\mathrm{~b}))$.

\subsection{Cathodoluminescence Spectral Features and Imaging Prop-} erties. In the area TLa, both chondrules have dull red color, but mesostasis of the barred part of the right-side chondrule has grayish green color (Figures $1(\mathrm{~d})$ and $1(\mathrm{f})$ ). It is important to note that these parts could also be trapped bits of diamond polishing compounds. The mesostasis has a number of red luminescent grains. On the backscattered electron (BSE) image the granular-barred chondrule has a lighter alteration rim including metal (Fe-Ni) and phyllosilicates (Figure 1(e)). The olivine grains in the chondrules show zoned higher luminescence (MiniCL image) according to the distribution of activator elements (Figure 1(b)). The alteration rims for both chondrules are composed of nonluminescent minerals (dominantly phyllosilicates). The dull luminescent grains have higher luminescent and nonluminescent rims. The change of luminescence intensity in adjacent grains is due to inhomogeneous distribution of CL activator elements. Inside the olivine, blue and light yellow inclusions occur.

$\mathrm{CL}$ area $\mathrm{b}$ (TLb) is composed primarily of strongly altered chondrule fragments (less than $300 \mu \mathrm{m}$ ) and isolated olivine fragments. The matrix is composed of phyllosilicates, carbonates, sulfides, magnetite, and organic material. Moreover, Izawa et al. [6] observed that the carbonate grains usually have a "streak" in the scanning electron microscope-cathodoluminescence (SEM-CL) images due to the long lifetime (phosphorescence rather than luminescence) (Figure 2(b)). Mineral fragments in the matrix have red luminescence color, whereas the nonaltered inner part of the chondrule has a blue 


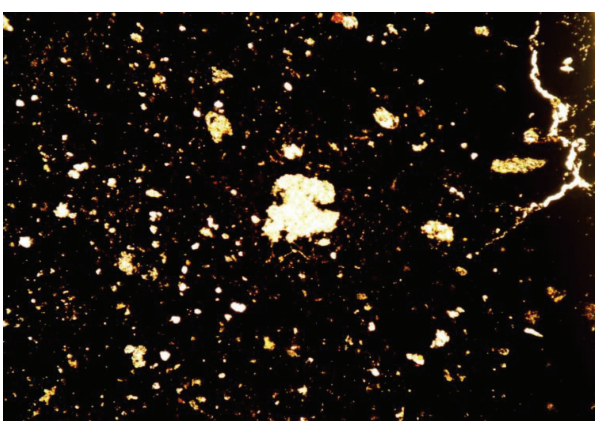

(a)

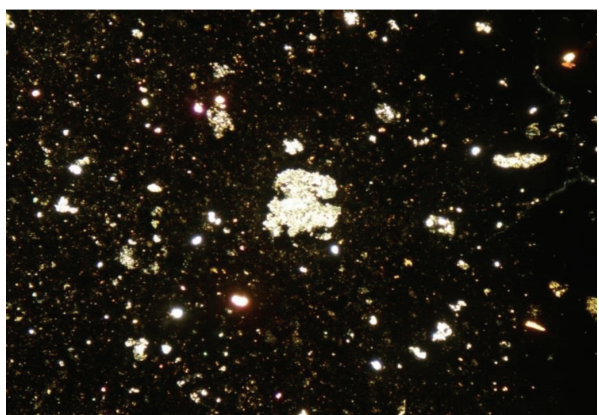

(c)

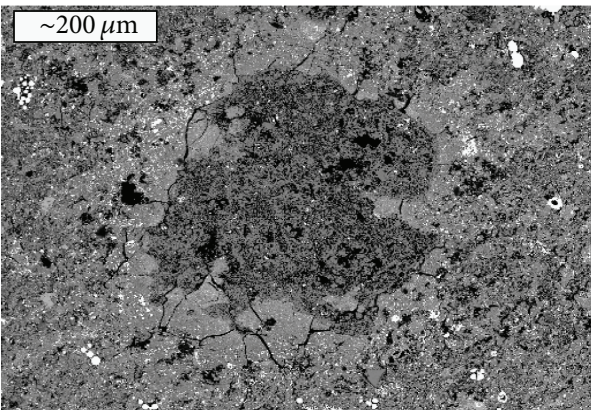

(e)

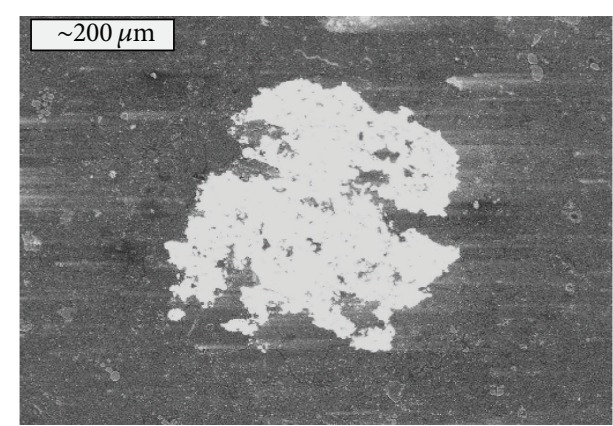

(b)

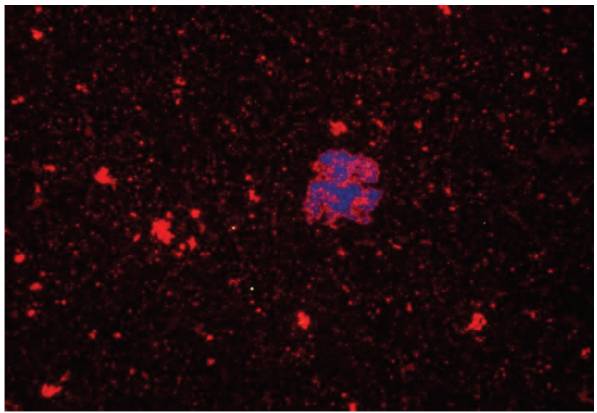

(d)

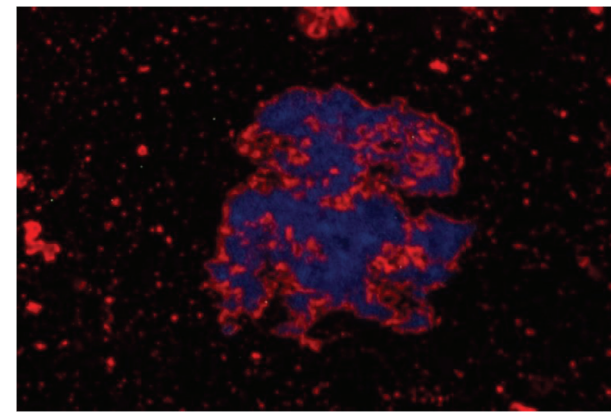

(f)

FIGURE 2: Optical microscope ((a) reflected light and (c) cross-polarized modes), scanning electron microscope ((e) backscattered electron microscope and (b) cathodoluminescence), and cathodoluminescence color ((d) and (f) at high magnification of the selected grain) images of TLb area showing a highly altered forsteritic chondrule. Scale: width of the chondrule is around $1 \mathrm{~mm}$.

luminescence color and red rim (Figures 2(d) and 2(f)). In the BSE image (Figure 2(e)), the inner part of the chondrule is dark and has a "spongy" texture. The alteration rim has lighter BSE contrast and contains metallic grains. The dark BSE indicates a predominance of low-Z elements like $\mathrm{Mg}, \mathrm{Si}$, and $\mathrm{O}$, consistent with forsterite [6]. The MiniCL image shows homogenous strong luminescence intensity for the whole inner part of the chondrule (Figure 2(b)).

The CL spectra show broad luminescence centers at 400$460,600-650$, and $700 \mathrm{~nm}$. TLb1 has less intensity in the $600-700 \mathrm{~nm}$ region than TLb2, while, in contrast to both TLb spectra, the TLa spectra have no luminescence center at $400 \mathrm{~nm}$. Instead, they have broad shoulders at 600-650, 700-720, and $750 \mathrm{~nm}$ (Figure 3(a)). After the peak fitting procedure (see Samples and Experimental Procedure), a peak at $400 \mathrm{~nm}$ (TLb1-2 only) and shoulders at 600-650 and 700$800 \mathrm{~nm}$ can be identified (Figure 3(b)). On the energy scale
(Figure 3(c)), broad shoulders occur at $0-2 \mathrm{eV}$, and a broad peak appears at $2.5-3.5 \mathrm{eV}$.

3.3. Raman Spectra. Raman spectral features (Figure 4) of the selected forsterite grain (TLb) contain several very weak (vw) as well as weak (w) positions at 222, 322, 390, 432, 541, 582, 605,668 , and $735 \mathrm{~cm}^{-1}$ and three medium strong peaks $(\mathrm{m})$ at 637,916 , and $963 \mathrm{~cm}^{-1}$. The spectrum is dominated by a very strong (vs) doublet peak at 823 and $855 \mathrm{~cm}^{-1}$ with a shoulder peak centered at $876 \mathrm{~cm}^{-1}$ (Figure 4).

\section{Discussion}

4.1. Cathodoluminescence Microscopy and Spectroscopy of Forsterite from Tagish Lake Meteorite. Cathodoluminescence properties of forsterite in meteorites were previously studied 


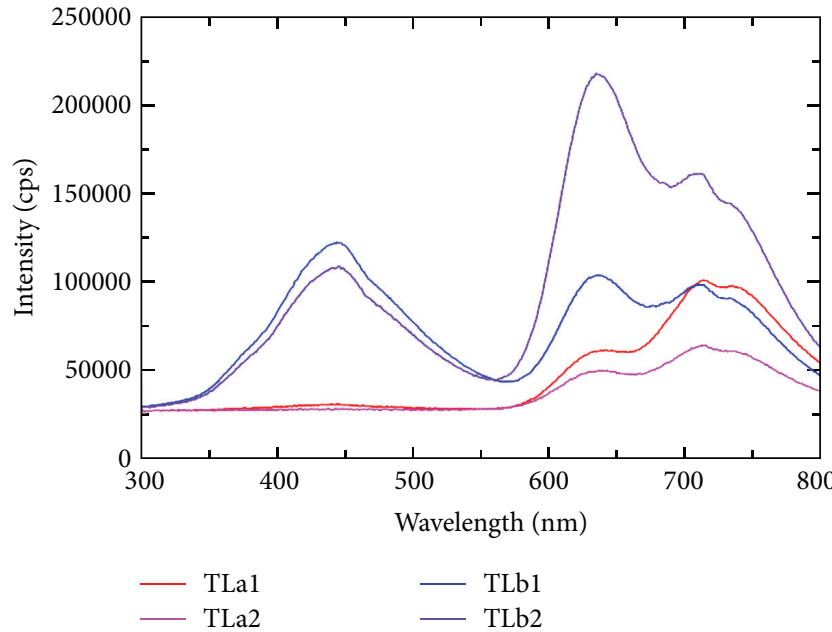

(a)

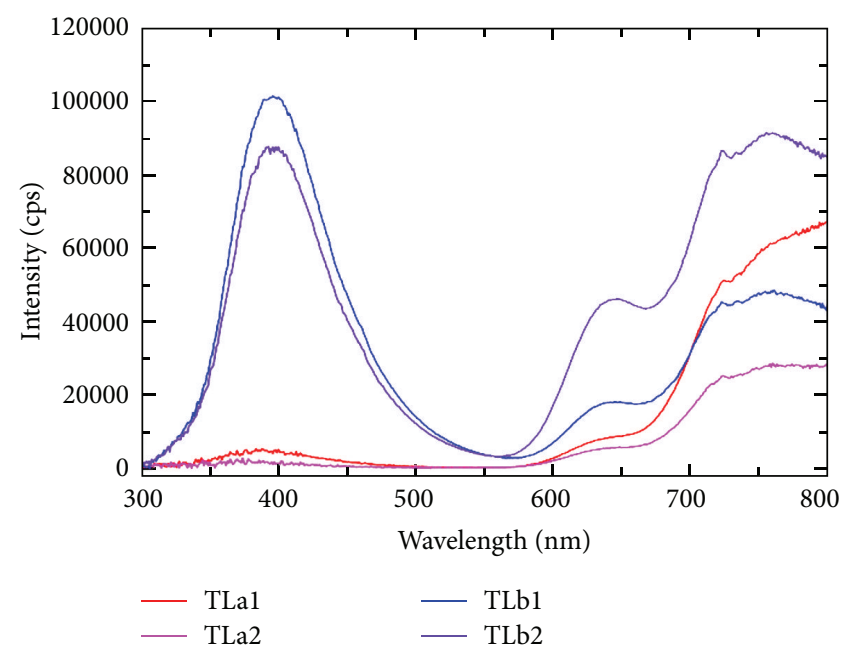

(b)

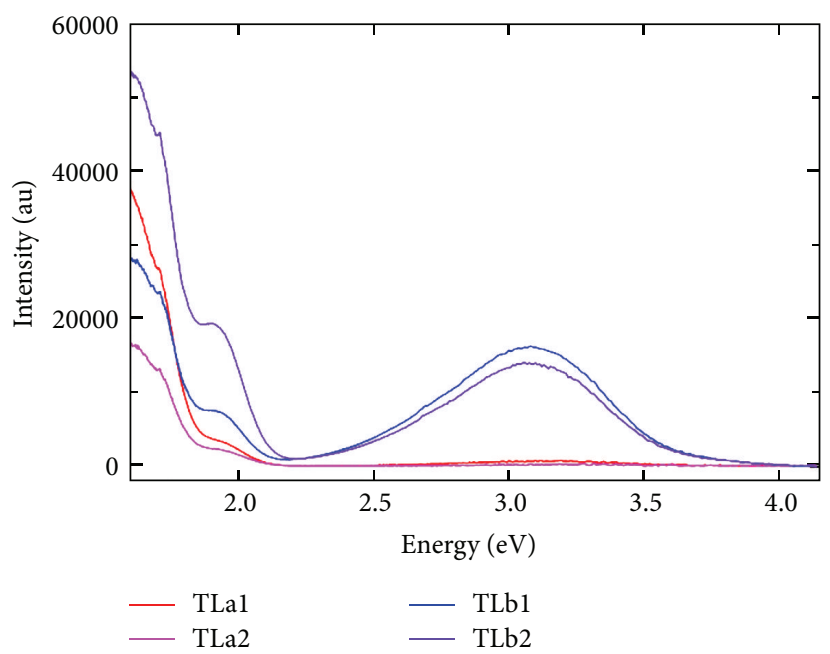

(c)

FIgURE 3: (a) Cathodoluminescence spectra of TLa and TLb areas in forsterite showing three major regions centered at 400-460, 600-650, and 700-800 nm. (b) CL spectra of forsterite grains followed by a peak fitting procedure. (c) CL intensity versus energy plot of the Tagish Lake forsterite. The $1.74 \mathrm{eV}$ peak corresponds to $\mathrm{Cr}^{3+}$, and the $1.94 \mathrm{eV}$ peak is assigned to $\mathrm{Mn}^{2+}$.

in the Kaba CV3 chondrite by Gucsik et al. [19, 20]. Similar to those, forsterite crystals in Tagish Lake show red-dull red luminescence. The lack of luminescence in fractures as well as decreased luminescence intensity in forsterite adjacent to fractures likely reflects increased concentration of $\mathrm{Fe}^{2+}$ in such regions, because of its quenching effect. The two broad centers at $630 \mathrm{~nm}$ in the red region and at $700-800 \mathrm{~nm}$ in the IR region can be caused by $\mathrm{Mn}^{2+}$ ion as activator, and by the $\mathrm{Cr}^{3+}$ activator, which may cause structural defects. The broad luminescence centered at $400 \mathrm{~nm}$ in case of the TLb area corresponds to a structural defect. Recently, Gucsik et al. [21] described a tendency that an increasing supercooling rate during the crystallization process of experimentally grown forsterite chondrules leads to a gradual cathodoluminescence color change ranging from red to greenish blue. They found that the red CL color corresponds to growth rates of 20100 micrometers/sec, whereas blue or greenish blue colors correspond to rates of $\sim 1000$ micrometers/sec. Nishido et al. [22] pointed out that CL-zoning records the thermal history of chondrules. During terrestrial weathering, $\mathrm{Fe}^{2+}$ cations are attached in the fractures resulting in quenching of luminescence. The unweathered meteoritic olivine (forsterite), on the other hand, is CL-active. The variation of luminescence intensity in chondrules of area A indicates chemical inhomogeneity due to low degree of thermal metamorphism. At the duller red luminescence centers of area TLa the olivine has a fayalitic component, whereas the light luminescent patches are purely forsterite. The fractures in chondrules are nonluminescent, which is caused by either enrichment of divalent Fe due to parent body aqueous alteration, terrestrial weathering, or shock-driven diffusion of $\mathrm{Fe}^{2+}$ into the olivine lattice. The blue luminescence center in area TLb is ascribed to intrinsic defects centers associated with either $\mathrm{Al}^{3+}$ substitution for $\mathrm{Si}^{4+}$ in tetrahedral sites or lattice deformation 


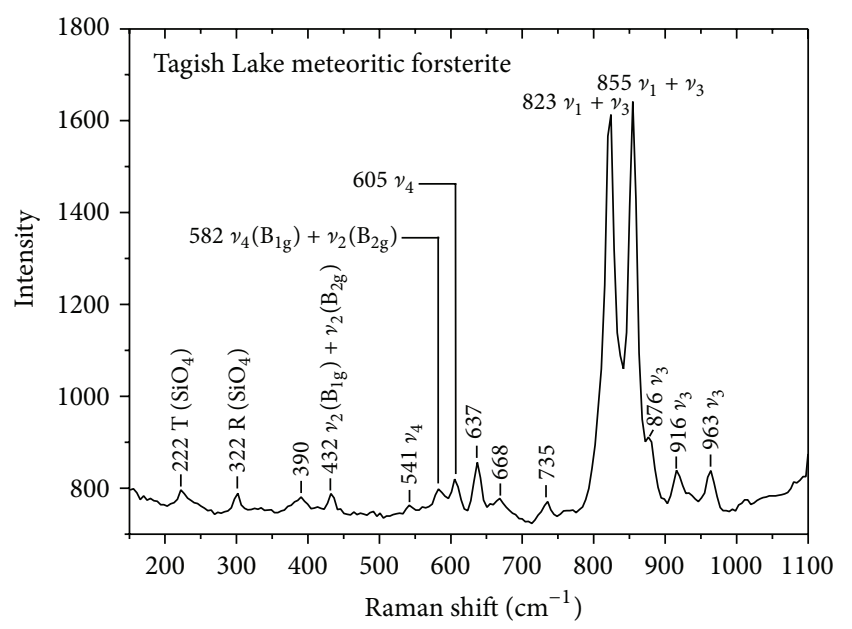

FIgURE 4: Raman spectrum of Tagish Lake forsterite showing a dominant doublet peak at 823 and $855 \mathrm{~cm}^{-1}$, which are assigned to symmetric and asymmetric stretching vibrational modes of the isolated $\mathrm{SiO}_{4}$ tetrahedra [18].

due to substitution of $\mathrm{Ca}^{2+}$ and $\mathrm{Ti}^{4+}$ ions in octahedral sites $[22,23]$. The broad emission at $650 \mathrm{~nm}$ (TLa, TLb) is assigned to $\mathrm{Mn}^{2+}$ impurity centers in $\mathrm{M} 2$ positions of forsterite [22, 23]. Finally, the broad emission bands at $720 \mathrm{~nm}$ and higher wavenumber is attributed to $\mathrm{Cr}^{3+}$ substitutions in the M1 and M2 sites, as well as interstitial positions of forsterite [22, 23].

The energetic CL spectra were deconvolved using the Mott-Seitz model [22]. This model obtains activation energies of temperature-quenching luminescence from a proposed nonradiative transition increasing with increasing sample temperature $[24,25]$. The activation energies for red emissions are centered at 1.8 and $1.74 \mathrm{eV}$ for TLa-b, where the $1.74 \mathrm{eV}$ peak corresponds to $\mathrm{Cr}^{3+}$ and the $1.94 \mathrm{eV}$ peak corresponds to $\mathrm{Mn}^{2+}$. The activation energy in blue region (TLb) appears as broad band at $3.15 \mathrm{eV}$, which corresponds to a crystallographic defect center, probably microdefect centers due to the rapid cooling history of forsterite [21] (Figure 3(b)).

4.2. Raman Spectroscopy. According to Chopelas [26] and Kolesov and Geiger [18] Raman properties of forsterite should be divided into two major spectral regions such as librational and translational modes as well as stretching vibrations of $\mathrm{SiO}_{4}$. In our study, two peaks centered at 222 and $322 \mathrm{~cm}^{-1}$ are assigned to translation $\left[\mathrm{T}\left(\mathrm{SiO}_{4}\right)\right]$ and liberation $\left[\mathrm{R}\left(\mathrm{SiO}_{4}\right)\right]$ of the $\mathrm{SiO}_{4}$. A peak at $432 \mathrm{~cm}^{-1}$ is a $v_{2}\left(\mathrm{~B}_{1 \mathrm{~g}}\right)+v_{2}\left(\mathrm{~B}_{2 \mathrm{~g}}\right)$ vibrational mode and a vibration at $582 \mathrm{~cm}^{-1}$ belongs to $v_{4}\left(\mathrm{~B}_{1 \mathrm{~g}}\right)+v_{2}\left(\mathrm{~B}_{2 \mathrm{~g}}\right)$. In our Tagish Lake meteoritic forsterite, there are two $v_{4}$ stretching modes centered at 541 and $605 \mathrm{~cm}^{-1}$. Peaks at 823 and $855 \mathrm{~cm}^{-1}$ are related to $v_{1}+v_{3}$ vibrational modes and a shoulder peak at 876 and two weak bands are assigned to $\nu_{3}$ stretching vibration. Four Raman spectral positions of the meteoritic forsterite centered at $390,637,668$, and $735 \mathrm{~cm}^{-1}$ are still poorly understood. Compared to other meteoritic forsterite (see $[19,27]$ and references therein), neither shock metamorphism nor thermal effects were found in the Raman properties of our Tagish Lake meteoritic forsterite. However, the Raman characteristics of our forsterite sample contains some signatures of the rapid cooling (e.g., Raman peaks centered at $390,637,668$, and $735 \mathrm{~cm}^{-1}$ ) [18], which are in a good agreement with the cathodoluminescence results of this study.

4.3. Forsterite Grains of Cometary Dust. It is known that the dust of comets in the form of silicate and carbon grains, as well as the fine-grained icy particles that make the cometary halo, causes the appearance of weak continuous spectrum of comets [28]. Recently forsterite mineral has been detected in the cometary dust [29]. It means that forsterite might be the noticeable component of the cometary silicate halos.

Cometary halos might be influenced by the fluxes of solar corpuscular radiation, solar wind, and plasma clouds. Solar flares might play an important role in the processes of interaction of radiation with the cometary atmospheres. The proton flares with outflows of protons with energy of more than $12 \mathrm{GeV}$ are rare phenomena. Proton flares with the energy of $10 \mathrm{MeV}<E<100 \mathrm{MeV}$ are more frequent. At the distance of $1 \mathrm{AU}$ from the Sun, the proton fluxes of the solar wind can vary within $10^{8}-10^{10} \mathrm{~cm}^{-2} \mathrm{~s}^{-1}$ [30]. Relativistic particles require not less than $8 \mathrm{~min}$ for propagation at the distance of $1 \mathrm{AU}$ and the electrons of $50 \mathrm{KeV}$ or ions of $100 \mathrm{MeV} \mathrm{amu}^{-1}$ require $18 \mathrm{~min}$ and for ions $1 \mathrm{MeV}$ amu $^{-1}$ they require 2.9 hours. For large and small solar flares the characteristic values of released energies can be expressed as $(3-5) \cdot 10^{31} \mathrm{erg}$. for electrons (of $20 \mathrm{KeV}$ and higher) and $(1-3) \cdot 10^{31} \mathrm{erg}$. for protons (of $20 \mathrm{MeV}$ and higher). Among the important phenomena is also the ejection of fast particles with velocity exceeding $1000 \mathrm{kms}^{-1}$. Taking into account the velocities of the main components of solar wind around $380 \mathrm{kms}^{-1}-800 \mathrm{kms}^{-1}$, it is necessary to note that the propagation of solar wind is limited by the dimensions of the heliosphere of about $100 \mathrm{AU}$ (astronomical unit). The release of a significant amount of energy during the solar flares takes place for a short period of time, in average, for tens of minutes. The fluxes of solar electrons and protons colliding with cometary halos might cause an intensive cathodoluminescence of the grains of halos. The intensity of cathodoluminescence of halos grains can be especially high after solar proton flares. When halos grains are bombarded by electrons or protons, its surface can start luminescing. The duration of cathodoluminescence of cometary grains might vary from some minutes to several hours. Too long exposure of cosmic luminophors to high-energy electrons (protons) can cause the luminophor destruction resulting in a full or partial loss of their luminescence properties. The practical detection of cathodoluminescence of cometary grains will depend on (1) quantum yield $(\ell)$ of cathodoluminescence of the matter of the given grains and (2) albedo $(A)$ of the given halos. Numerically, for the case of cathodoluminescence $l \geq$ $40 \%$ and $A \leq 0.3$ will be favorable for detection from ground based telescopes.

Forsterite, quartz, and some other minerals are luminescing in red and blue spectral regions under the action of the fluxes of electrons. The spectra of luminescence of these 
minerals are often characterised by wide structureless bands in the red region with a peak near $600 \mathrm{~nm}$.

We have obtained CL spectra of Tagish Lake forsterite with maximum near 440 and $650 \mathrm{~nm}$. CL spectra of this forsterite are characterised in featureless nature with two main bumps. When the comet approaches the Sun, its electromagnetic and corpuscular radiation could excite intense luminescence of the cometary halo, including cathodoluminescence of the forsterite grains. CL luminescence of such forsterite particles may also be characterised in featureless emissions in blue and red parts of the spectrum. Such luminescence emissions will be superimposed on the faint solar continuum scattered by the cometary grains. The active cometary phenomena including outbursts and flares are characterised in release of the inner substance of cometary nuclei (relict dust and relict ice). Among the relicts might be pristine forsterite grains with the different sizes and shapes. The spectra of cometary outbursts have in many cases featureless character especially in blue range (maxima within 440-490 nm). Investigation of the cometary spectra obtained in time of outbursts might be as the effective tool for revealing of pristine cometary substance including presolar forsterite. Laboratory data of CL of Tagish Lake forsterite (obtained by us) might be suitable comparative sources in investigation of the cometary dust (cometary astromineralogy). It will be especially useful for revealing of presolar minerals of cometary substance.

\section{Conclusions}

(i) The CL zonation in Tagish Lake forsterite reflects rapid cooling and crystallization of the chondrule melts, preserving both structural defects (responsible for the blue CL emission) and inhomogeneities of activator elements, as well as possible remobilization of activators by later fluid processes on the parent asteroid.

(ii) We conclude that a combination of scanning electron microscope-cathodoluminescence and Raman spectroscopy would be a powerful tool to understand not only the asteroidal processes but also the astromineralogical aspects of crystallization in the early solar system.

(iii) Moreover, these techniques would be applied to the mineralogical investigations for the future samplereturn missions, too.

\section{Conflict of Interests}

The authors declare that there is no conflict of interests regarding the publication of this paper.

\section{Acknowledgments}

Matthew M. R. Izawa acknowledges funding from NSERC and the Mineralogical Association of Canada, Arnold Gucsik was partly supported by the NRF Free-Standing Fellowship Program at the Department of Geology, University of Johannesburg, South Africa. Authors are grateful to Dr. Phil
McCausland (University of Western Ontario, Canada) for lending the Tagish Lake meteorite thin section for this study.

\section{References}

[1] P. G. Brown, A. R. Hildebrand, M. E. Zolensky et al., "The fall, recovery, orbit, and composition of the Tagish Lake meteorite: a new type of carbonaceous chondrite," Science, vol. 290, no. 5490, pp. 320-325, 2000.

[2] P. G. Brown, D. O. ReVelle, E. Tagliaferri, and A. R. Hildebrand, "An entry model for the Tagish Lake fireball using seismic, satellite and infrasound records," Meteoritics and Planetary Science, vol. 37, no. 5, pp. 661-675, 2002.

[3] A. R. Hildebrand, P. J. A. McCausland, P. G. Brown et al., "The fall and recovery of the Tagish Lake meteorite," Meteoritics and Planetary Science, vol. 41, no. 3, pp. 407-431, 2006.

[4] M. M. Grady, A. B. Verchovsky, I. A. Franchi, I. P. Wright, and C. T. Pillinger, "Light element geochemistry of the Tagish Lake $\mathrm{C} 12$ chondrite: comparison with CI1 and CM2 meteorites," Meteoritics and Planetary Science, vol. 37, no. 5, pp. 713-735, 2002.

[5] M. E. Zolensky, K. Nakamura, M. Gounelle et al., "Mineralogy of Tagish Lake: an ungrouped type 2 carbonaceous chondrite," Meteoritics and Planetary Science, vol. 37, no. 5, pp. 737-761, 2002.

[6] M. R. M. Izawa, R. L. Flemming, P. J. A. McCausland, G. Southam, D. E. Moser, and I. R. Barker, "Multi-technique investigation reveals new mineral, chemical, and textural heterogeneity in the Tagish Lake C2 chondrite," Planetary and Space Science, vol. 58, no. 10, pp. 1347-1364, 2010.

[7] M. R. M. Izawa, R. L. Flemming, P. L. King, R. C. Peterson, and P. J. A. McCausland, "Mineralogical and spectroscopic investigation of the Tagish Lake carbonaceous chondrite by X-ray diffraction and infrared reflectance spectroscopy," Meteoritics and Planetary Science, vol. 45, no. 4, pp. 675-698, 2010.

[8] A. I. Blinova, C. D. K. Herd, and M. J. M. Duke, “Testing variations within the Tagish Lake meteorite-II: whole-rock geochemistry of pristine samples," Meteoritics and Planetary Science, vol. 49, no. 6, pp. 1100-1118, 2014.

[9] A. I. Blinova, T. J. Zega, C. D. K. Herd, and R. M. Stroud, “Testing variations within the Tagish Lake meteorite-I: mineralogy and petrology of pristine samples," Meteoritics and Planetary Science, vol. 49, no. 4, pp. 473-502, 2014.

[10] C. D. K. Herd, A. Blinova, D. N. Simkus et al., "Origin and evolution of prebiotic organic matter as inferred from the Tagish Lake meteorite," Science, vol. 332, no. 6035, pp. 1304-1307, 2011.

[11] E. R. D. Scott, K. Keil, and D. Stöffler, "Shock metamorphism of carbonaceous chondrites," Geochimica et Cosmochimica Acta, vol. 56, no. 12, pp. 4281-4293, 1992.

[12] T. Hiroi, M. E. Zolensky, and C. M. Pieters, "The Tagish Lake meteorite: a possible sample from a D-type asteroid," Science, vol. 293, no. 5538, pp. 2234-2236, 2001.

[13] D. W. Mittlefehldt, "Geochemistry of the ungrouped carbonaceous chondrite Tagish Lake, the anomalous CM chondrite Bells, and comparison with CI and CM chondrites," Meteoritics and Planetary Science, vol. 37, no. 5, pp. 703-712, 2002.

[14] S. Pizzarello, Y. Huang, L. Becker et al., "The organic content of the Tagish Lake meteorite," Science, vol. 293, no. 5538, pp. 22362239, 2001.

[15] K. Nakamura-Messenger, S. Messenger, L. P. Keller, S. J. Clemett, and M. E. Zolensky, "Organic globules in the Tagish Lake 
meteorite: Remnants of the protosolar disk," Science, vol. 314, no. 5804, pp. 1439-1442, 2006.

[16] S. D. J. Russell, F. J. Longstaffe, P. L. King, and T. E. Larson, "The oxygen-isotope composition of chondrules and isolated forsterite and olivine grains from the Tagish Lake carbonaceous chondrite," Geochimica et Cosmochimica Acta, vol. 74, no. 8, pp. 2484-2499, 2010.

[17] M. Kayama, S. Nakano, and H. Nishido, "Characteristics of emission centers in alkali feldspar: a new approach by using cathodoluminescence spectral deconvolution," American Mineralogist, vol. 95, no. 11-12, pp. 1783-1795, 2010.

[18] B. A. Kolesov and C. A. Geiger, "A Raman spectroscopic study of Fe-Mg olivines," Physics and Chemistry of Minerals, vol. 31, no. 3, pp. 142-154, 2004.

[19] A. Gucsik, T. Endo, E. Nakazato et al., "Cathodoluminescence characterization of the forsterite in Kaba meteorite: an astromineralogical application," in Proceedings of the 42nd Lunar and Planetary Science Conference, abs\#1157, The Woodlands, Tex, USA, March 2011.

[20] A. Gucsik, T. Endo, H. Nishido et al., "Cathodoluminescence microscopy and spectroscopy of forsterite from Kaba meteorite: an application to the study of hydrothermal alteration of parent body," Meteoritics and Planetary Science, vol. 48, no. 12, pp. 2577-2596, 2013.

[21] A. Gucsik, K. Tsukamoto, H. Nishido et al., "Cathodoluminescence microcharacterization of forsterite in the chondrule experimentally grown under super cooling," Journal of Luminescence, vol. 132, no. 4, pp. 1041-1047, 2012.

[22] H. Nishido, T. Endo, K. Ninagawa, M. Kayama, and A. Gucsik, "Thermal effects on cathodoluminescence in forsterite," Geochronometria, vol. 40, no. 4, pp. 239-243, 2013.

[23] E. J. Benstock, P. R. Buseck, and I. M. Steele, "Cathodoluminescence of meteoritic and synthetic forsterite at 296 and $77 \mathrm{~K}$ using TEM," American Mineralogist, vol. 82, no. 3-4, pp. 310-315, 1997.

[24] F. Seitz, "An interpretation of crystal luminescence," Transactions of the Faraday Society, vol. 35, pp. 74-85, 1939.

[25] N. F. Mott and R. W. Gurney, Electronic Processes in Ionic Crystals, Clarendron Press, Oxford, UK, 1948.

[26] A. Chopelas, "Single crystal Raman spectra of forsterite, fayalite, and monticellite," American Mineralogist, vol. 76, no. 7-8, pp. 1101-1109, 1991.

[27] F. Rull, M. J. Muñoz-Espadas, R. Lunar, and J. MartínezFrías, "Raman spectroscopic study of four Spanish shocked ordinary chondrites: Cañellas, Olmedilla de Alarcón, Reliegos and Olivenza," Philosophical Transactions of the Royal Society A: Mathematical, Physical and Engineering Sciences, vol. 368, no. 1922, pp. 3153-3166, 2010.

[28] I. Simonia, "On the possible luminescence nature of unidentified cometary emissions," Astrophysics and Space Science, vol. 312, no. 1-2, pp. 27-33, 2007.

[29] D. S. Lauretta, L. P. Keller, and S. Messenger, "Supernova olivine from cometary dust," Science, vol. 309, no. 5735, pp. 737-741, 2005.

[30] R. Noyes, The Sun: Our Star, Harvard University Press, Cambridge, Mass, USA, 1983. 

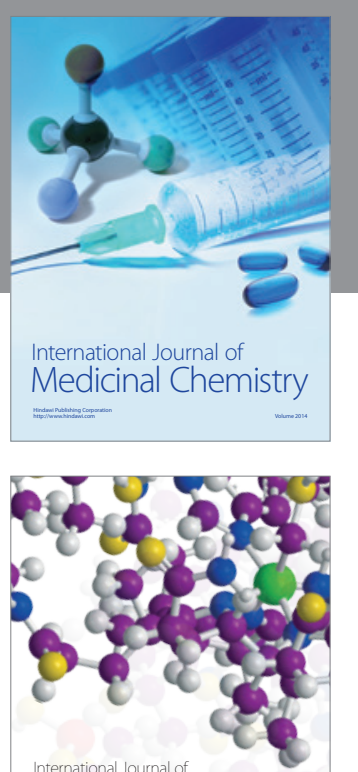

Carbohydrate Chemistry

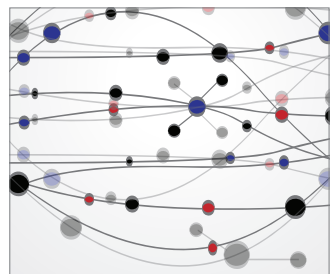

The Scientific World Journal
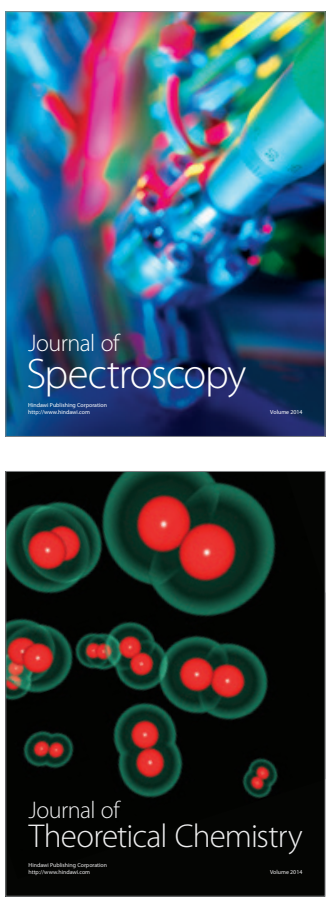
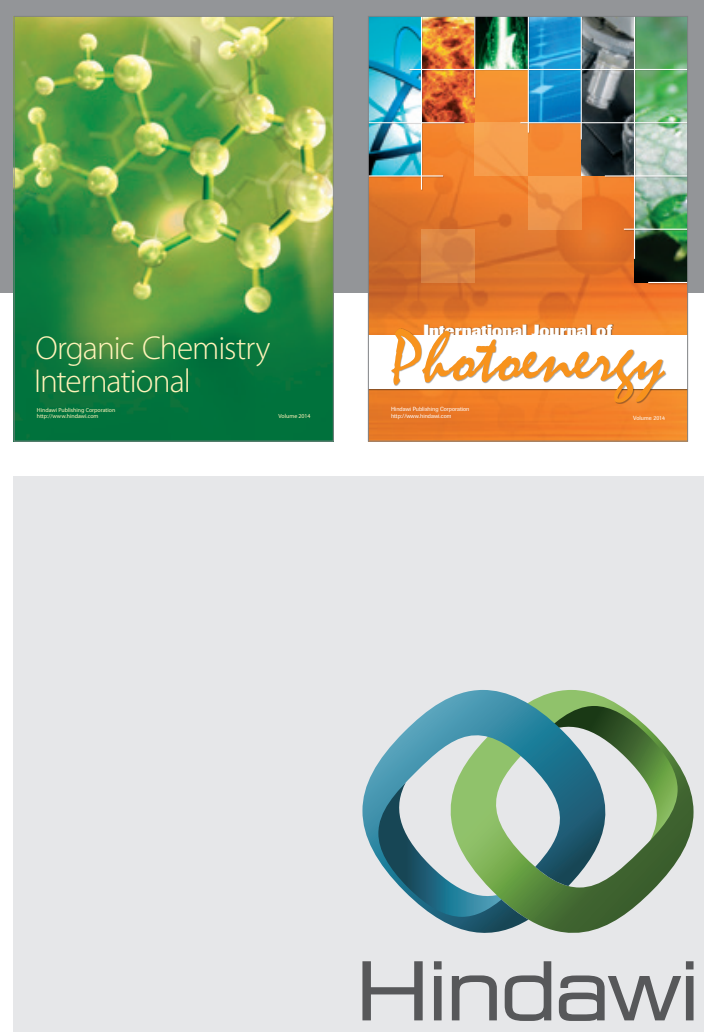

Submit your manuscripts at

http://www.hindawi.com

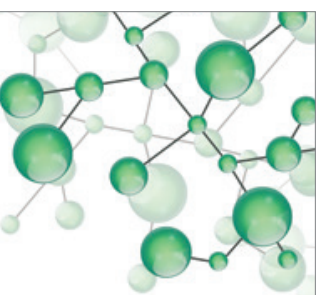

International Journal of

Inorganic Chemistry

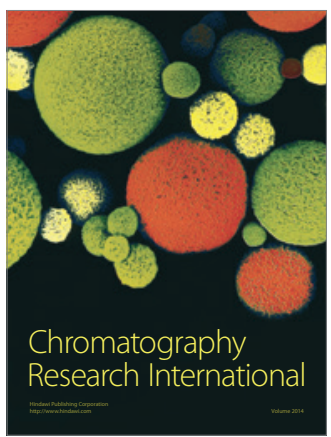

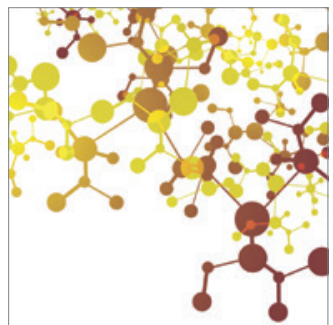

Applied Chemistry
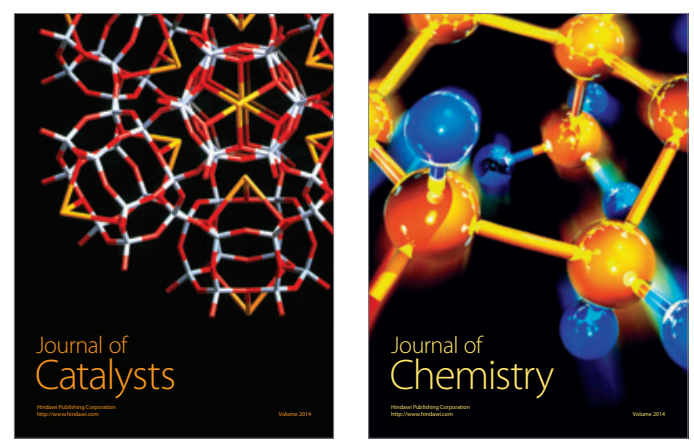
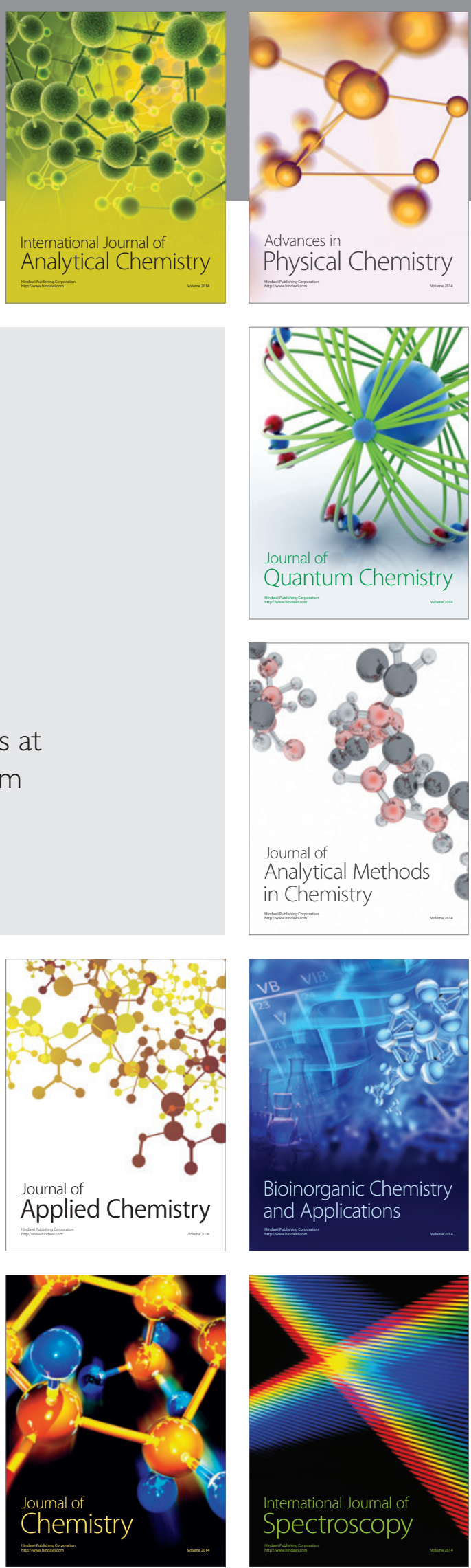